\title{
Isolation and Identification of Potential Zoonotic Dermatophytes from Domestic Camels in Dhamar Area, Yemen
}

\author{
Najla M. Baghza ${ }^{1,}$, , Abdulelah H. Al-Adhroey ${ }^{2}$, Abdullatif D. Ali ${ }^{3}$ \\ ${ }^{1}$ Department of Microbiology, Faculty of Medicine and Health Sciences, Thamar University, Dhamar, Yemen \\ ${ }^{2}$ Department of Medical Basic Sciences, Faculty of Medicine and Health Sciences, Thamar University, Dhamar, Yemen \\ ${ }^{3}$ Department of Biochemistry, Faculty of Medicine and Health Sciences, Thamar University, Dhamar, Yemen
}

Email address:

baghza2006@yahoo.com (N. M. Baghza), husssien75@gmail.com (A. H. Al-Adhroey), abdullatifwhite@gmail.com (A. D. Ali)

\section{To cite this article:}

Najla M. Baghza, Abdulelah H. Al-Adhroey, Abdullatif D. Ali. Isolation and Identification of Potential Zoonotic Dermatophytes from Domestic Camels in Dhamar Area, Yemen. American Journal of Health Research. Vol. 4, No. 3, 2016, pp. 46-50. doi: 10.11648/j.ajhr.20160403.12

Received: April 20, 2016; Accepted: April 28, 2016; Published: May 11, 2016

\begin{abstract}
Dermatophytosis is a prevalent skin disease in Yemen. Because it's zoonotic transmission to humans, animal dermatophytosis has a major concern. The present study was thus aimed to isolate and identify dermatophytes from domestic camels. A purposive study was conducted among 165 suspected camels in Dhamar area, Yemen. Skin scrapings were collected from ringworm lesions of the study animals. They were microscopically examined using Potassium Hydroxide (KOH) preparation. Specimens that showed a positive result were then cultured on Sabouraud's Dextrose Agar (SDA) for identification. A total of 159 (96.4\%) of the suspected camels were found to be infected with fungal infection during the direct $\mathrm{KOH}$ examination. The infection was significantly higher among young animals of $\leq 12$ months $\left(94.3 \%, \chi^{2}=73, P<0.05\right)$. Majority of the cultured specimens showed positive growth $\left(93.1 \%, \chi^{2}=118, P<0.05\right)$. The overall rate of dermatophyte infection was $83.11 \%$. The frequency of Trichophyton and Microsporum genera were $89.4 \%$ and $10.6 \%$ of the isolated genera, respectively. The identified species were T. schoenlenii T. verrucosum, T. mentagrophytes, T. tonsurans, M. audouinii and M. canis. Almost half of the study animals were infected with $T$. schoenlenii $\left(49.6 \%, \chi^{2}=102, P<0.05\right)$. Findings of the present study are important in order to provide the health authorities with an epidemiological and microbiological database that are vital for supporting well-organized control programs. Introduction of proper health education is important to improve prevention measures and introduce a better knowledge of dermatophytosis transmission.
\end{abstract}

Keywords: Dermatophytosis, Zoonotic Infection, Dhamar, Yemen

\section{Introduction}

Dermatophytosis (syn. ringworm, tinea) is a zoonotic disease caused by a group of fungi called dermatophytes. They invade keratinized tissues of animals causing losses to the milk and meat economy. Dermatophytosis has a worldwide distribution. It is the most frequent mycotic infection in both humans and animals [1].

Epidermophyton, Microsporum and Trichophyton are the main genera of dermatophytes. Based on their ecology, they are classified into anthropophilic, geophilic and zoophilic. They can directly infect human through a contact with the infested animal or human and indirectly by acquiring the infective materials from the environment [2]. The most common carriers of these microorganisms are particularly domestic animals such as cow, goat, sheep and camel [3]. Camel's long-haired skin presents a proper habitat for the growth of dermatophytes [5]. The infection mainly occurs in young camels less than three years [6].

Trichophyton and Microsporum genera are responsible for the majority of human infections causing more virulent disease when the infection are transmitted from animal to human than that transmitted from human to human [7-8]. Outbreaks of Trichophyton infection transmitted from cattle to humans are also reported [9-10].

In Yemen, human dermatopytosis is a public health problem [11-12]. Trichophyton verrucosum is reported as the major cause 
of cattle dermatophytosis in Dhamar area [13]. Dhamar is mostly a rural area where domestic animals represent cardinal components of the agriculture and economy. While reports have revealed that domestic cattle are often potential carriers of dermatophytes in this area, little information has been documented about domestic camels. In fact, to date there are neither epidemiological nor microbiological data about isolation and identification of dermatophytes from camels. Given the significant zoonotic transmission and pathogenicity of dermatophytes in humans, this study was thus aimed to isolate and identify dermatophytes from domestic camels in Dhamar area.

\section{Methodology}

\subsection{Study Area and Animals}

The present study was conducted in Dhamar area, 100 kilometers south of the capital city of Yemen, Sana'a "Fig. 1". It is located at $14^{\circ} 33^{\prime} 0^{\prime \prime} \mathrm{N} 44^{\circ} 24^{\prime} 6^{\prime \prime} \mathrm{E}$, at an altitude of around 2400 meters above sea level, and temperature between - 1 to $28^{\circ} \mathrm{C}$. A total of 165 suspected camels were selected from suburban and rural areas around Dhamar city. Sampling was at houses or farms during the period of March to August 2014. The body of each camel was carefully examined for indications of ringworm. Animal with a circumscribed crusty hairless lesion were included in this study.

During the field study, educational meetings were held with the owners of the study animals and clear descriptions about the objectives of the survey were presented. They were informed that they are free to participate and their animal's contribution is completely voluntary. All the owners were consented and the animals were ethically examined.

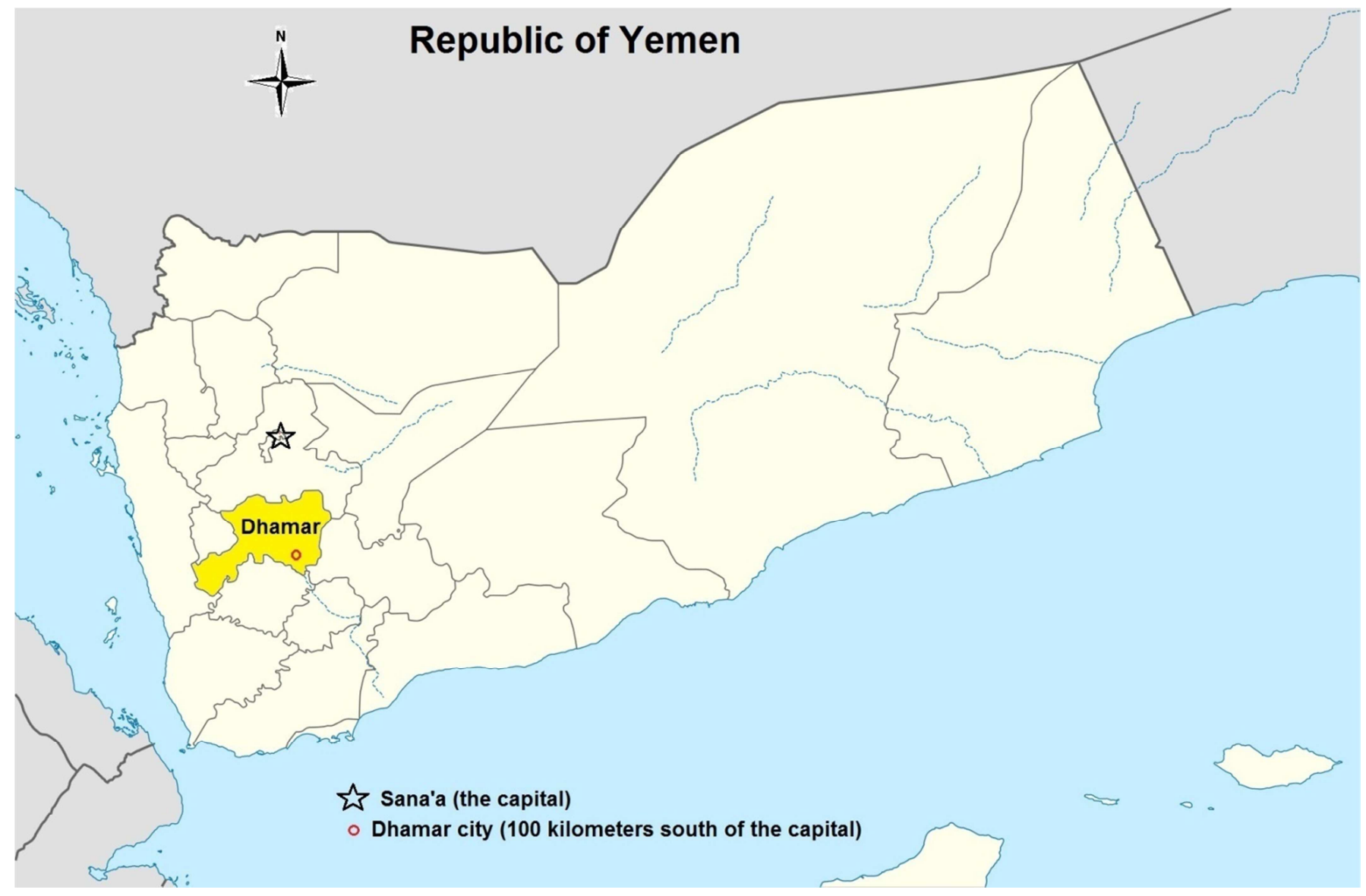

Figure 1. A geographic map shows Dhamar area (the study area) in Yemen.

\subsection{Sample Collection}

Cotton swabs, soaked in $70 \%$ ethanol, were used to clean the infected area. Lesions were scrapped by a sterile scalpel and collected in sterile petri-dishes. All specimens were then labeled for age, location, and date of collection. They were then transferred to the laboratory.

\subsection{Direct KOH Examination}

On a clean glass slide, a part of each animals sample was placed. Twenty percent (20\%) of Potassium Hydroxide (KOH) was used to digest the keratin material. The solution was then covered with a clean glass and gently heated for one minute. The slide was microscopically examined for the presence of arthrospores [14].

\subsection{Isolation of Dermatophytes}

To isolate dermatophytes, skin scrapings were cultured on Sabouraud's dextrose agar (SDA) containing chloramphenicol $(0.05 \mathrm{mg} / \mathrm{ml})$ and cyclohexamide $(0.5 \mathrm{mg} / \mathrm{ml})$. The inoculated 
plates were incubated at $27^{\circ} \mathrm{C}$ for up to six weeks. They were regularly observed for differentiated colonies. Growth rate, obverse, reverse pigmentation of the recovered colonies were reported [1].

\subsection{Identification of Dermatophytes}

Identification of dermatophytes was based on the colonies characteristic and microscopic features of the isolates. A small sample of each fungal mycelium free of the medium was examined using $10 \mathrm{X}$ and $40 \mathrm{X}$ objectives. The mycological text books used for identification were Laboratory Handbook of Dermatophytes, Veterinary Mycology Laboratory Manual and Identification of Common Aspergillus Species [1, 15-16].

\subsection{Statistical Analysis}

SPSS version 22.0 statistical software was used in this study. For the purpose of data analysis, all the factors in the study were coded in a characteristic manner. For example, $\mathrm{KOH}$ results (positive, negative); age groups (2-5 months, 6-12 months, $>12$ months); culture results (positive growth, negative growth); isolated fungi (dermatophytes, non-dermatophytes); identified genera (Trichophyton, Microsporum). All the study groups were presented as a frequency (\%). Chi-square test $\left(\chi^{2}\right)$ was used to examine the differences between the study groups. The level of significant was of $P \leq 0.05$.

\section{Results}

\subsection{General Characteristics of Animal's Infection}

Table 1 shows the general characteristics of the study camels' infection. The study population consisted of 165 domestic camels. Fungal infection was confirmed in $96.4 \%$ $(159 / 165)$. The remaining $(3.6 \%)$ were $\mathrm{KOH}$ negative and they did not yield any fungal growth on SDA agar as well.

Fungal infections were found to be significantly $(P<0.001)$ associated with age of the study subjects; younger animals had a higher rate of infection. Sixty one percent $(61 \%)$ of the infected camels were in the age group of two to five months. As well, one third $(33.3 \%)$ of the infections were reported from the age group of six to 12 months. However, camels aged $>12$ months represented only $5.7 \%$ of the infected subjects.

\subsection{Isolated Fungi}

Out of the 159 positive $\mathrm{KOH}$ specimens, fungal growth was recovered from 148 SDA plates $\left(93.1 \%, \chi^{2}=118, P<0.05\right)$. Species-specific colonies were successfully recognized. The majority of the isolated species were established for dermatophytes (83.1\%). However, non-dermatophytes species were identified for $16.89 \%$ of the cultured specimens.

\subsection{Identified Dermatophytes}

Two genera of dermatophytes were identified among camels in Dhamar area: Trichophyton and Microsporum.

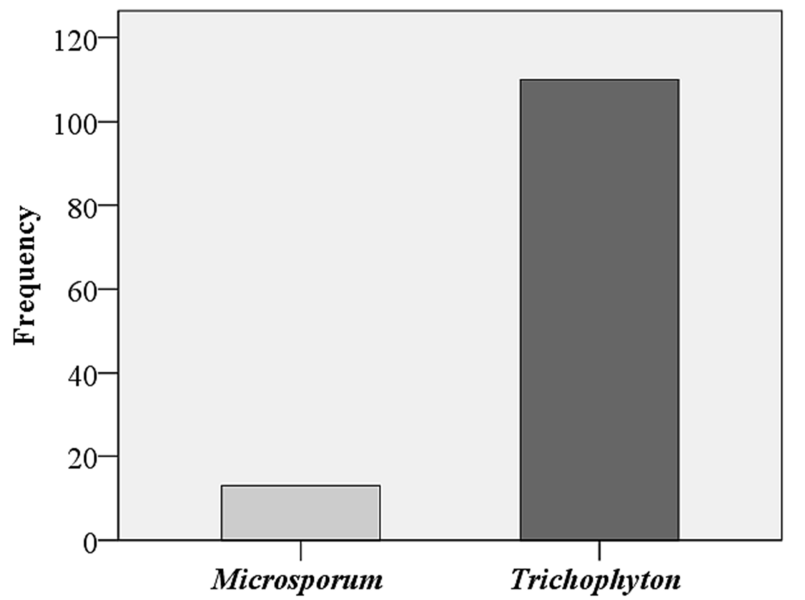

Figure 2. Frequency of Trichophyton and Microsporum genera isolated from the domestic camels in Dhamar area, Yemen.

Table 1. General characteristics of the study camels' infection in Dhamar area, Yemen ( $n=165)$.

\begin{tabular}{|c|c|c|c|}
\hline Characteristics & Frequency $(\%)$ & $\chi^{2}$ & $P$ \\
\hline \multicolumn{4}{|l|}{ KOH results $(n=165)$} \\
\hline Positive fungal elements & $159(96.4)$ & 141.87 & $<0.05$ \\
\hline Negative fungal elements & $6(3.6)$ & & \\
\hline \multicolumn{4}{|c|}{ Age groups of the infected camels $(n=159)$} \\
\hline $2-5$ months & $97(61.0)$ & 73.06 & $<0.05$ \\
\hline $6-12$ months & $53(33.3)$ & & \\
\hline \multicolumn{4}{|l|}{ Culture growth $(\mathrm{n}=159)$} \\
\hline Positive growth & $148(93.1)$ & 118.04 & $<0.05$ \\
\hline Negative growth & $11(6.9)$ & & \\
\hline \multicolumn{4}{|l|}{ Isolated fungi $(\mathrm{n}=148)$} \\
\hline Dermatophytes & $123(83.11)$ & 64.89 & $<0.05$ \\
\hline Non-dermatophytes & $25(16.89)$ & & \\
\hline
\end{tabular}

Figure 2 shows that the frequency of Trichophyton infection was considerably higher than Microsporum $(89.4 \%$ versus $\left.10.6 \% ; \chi^{2}=76.50 ; P<0.05\right)$.

\subsection{Identified Species}

As shown in Table 2, six dermatophytes were identified. The study animals were significantly infected with different 
species $\left(\chi^{2}=101.93 ; P<0.05\right)$. Trichophyton schoenlenii showed the highest frequency $(49.6 \%)$ followed by Trichophyton verrucosum (14.6\%), T. mentagrophytes (13\%) and Trichophyton tonsurans (12.2\%). On the other hand, Microsporum audouinii and Microsporum canis represented $5.7 \%$ and $4.9 \%$ of the identified species.

Table 2. Identified dermatophytes from domestic camels in Dhamar area, Yemen ( $n=123)$.

\begin{tabular}{llll}
\hline Isolated species & Frequency (\%) & $\chi^{2}$ & $\boldsymbol{P}$ \\
\hline Trichophyton schoenlenii & $61(49.6)$ & 101.93 & $<0.05$ \\
Trichophyton verrucosum & $18(14.6)$ & & \\
Trichophyton mentagrophytes & $16(13.0)$ & & \\
Trichophyton tonsurans & $15(12.2)$ & & \\
Microsporum audouinii & $7(5.7)$ & & \\
Microsporum canis & $6(4.9)$ & & \\
\hline
\end{tabular}

\section{Discussion}

Participation of the community is a principal tool for operating an integrated control methodology against the infectious diseases. In Yemen, dermatophytosis is an important skin problem affecting human population. Involvement of the domestic animals in acquiring this infection has complicated its control. Hence, community's awareness on transmission of this infectious disease would enhance the prevention and significantly supports the sustainability of control program.

The present study is the first to isolate human potential dermatophytosis causative fungi from domestic camels in Dhamar area. There was a significant difference between the age groups of animals in terms of their susceptibility to the infection. The results showed that the majority of the study animals had a dermatophytic infection. Six dermatophytes species were identified from domestic camel's infection in Dhamar area, Yemen: T. schoenlenii T. verrucosum, $T$. mentagrophytes, T. tonsurans, $M$. audouinii and M. canis.

The findings of this study found that Trichophyton fungi were responsible for the majority of camel's infection. Accordingly, T. schoenlenii, reported as one of the causative agents of camel dermatophytosis, was the common identified species in this study [17]. This microorganism has been widely confirmed to produce a severe form of tinea capitis in humans called favus that usually affects the scalp hair [18-20]. A previous study from Nigeria indicated that $T$. schoenlenii infection is more prevalent among the school children in Borno State compared to the other dermatophytic infections [21]. As well, T. mentagrophytes and T. verrucosum have been indicated as common causes of onychomycosis and ringworm infection in human, respectively [22]. In agreement with previous studies, T. mentagrophytes, T. verrucosum and $T$. tonsurans have been isolated from Sudanese and Saudi camels $[6,23]$. Also, T. mentagrophytes, T. verrucosum have been reported from school children in Yemen [24]. Although epidemiology of $T$. tonsurans in Yemen has not been documented in literature, previous studies confirmed its responsibility for about $90 \%$ of tinea capitis in North and Central America [25]. On the other hand, $M$. audouinii and $M$. canis has been commonly isolated from human dermatophytosis of tinea corporis and tinea capitis [26-27]. $M$. canis as well as $T$. violaceum were commonly isolated from Yemeni patients [24]. Similarly, camel dermal mycoses caused by $M$. audouinii and $M$. canis have been well documented [28]. Awareness of health providers regarding this situation is important in order to increase the preventive measures for healthy protection for domestic camels and the farmers who frequently contact them.

The present study also revealed that young camels were more infected than adults. This could be attributed to a stronger immunity among adults that acquired through repeated contacts or due to a proper microclimate of young camel's skin to dermatophytic infection [29]. Therefore, the contact with asymptomatic adult camels can represent a high risk factor of infection for the young camels and to humans. Humans may be infected through a direct contact with the infected camel or indirectly with contaminated materials. This highlights the need for introduction a health education to aware the community about the potential risk factors and mode of transmission of the causative agents.

\section{Conclusion}

The present study was designed to isolate the causative fungi of dermatophytosis from the domestic camels. That is aimed to elicit a public awareness headed for controlling this prevalent infectious disease in Dhamar area, Yemen. This survey revealed that the dermatophyte infection is common among the domestic camels. Hence, control program should take into account that health education will improve prevention and introduce a better awareness of dermatophytosis transmission. The results of this study would provide the health authorities with a mycological database that are essential for supporting well-organized tools for health education, sustaining effective methods for dermatophytosis prevention and establishing a literature for further epidemiological studies.

\section{Acknowledgements}

The authors would like to thank Dr. Ahmed Y. Saif from Sana'a Veterinary Institute for his great support during this study. We also thankfully appreciate the owners of camels for their kind help in the collection of the study samples.

\section{References}

[1] J. Kane, R. Summerbell, L. Sigler, S. Krajden, and G. Land, Laboratory Handbook of Dermatophytes. Belmont, CA, USA: Star Publishing Company, 1997.

[2] B. Hainer, "Dermatophyte infections", A. F. P., vol. 67, pp. 101-108, 2003. 
[3] M. Shams-Ghahfarokhi, F. Mosleh-Tehrani, S. Ranjbar-Bahadori, and M. Razzaghi-Abyaneh, "An epidemiological survey on cattle ringworm in major dairy farms of Mashhad city, Eastern Iran," Indi. Jour. of Microbio., vol. 1, pp. 31-36, 2009.

[4] Z. Baranova, M. Kozak, J. Bilek, and Z. Pomorski, "Zoophilic dermatomycosis in a family caused by Trichophyton mentagrophytes var. quinckeanum," Vet. Brno., vol. 72, pp. 311-314, 2003.

[5] H. Agab, Epidemiology of Camel Diseases in Eastern Sudan with Emphasis on Brucellosis, MSc Thesis. Sudan: University of Khartoum, 1993.

[6] A. G. Wisal, and M. O. Salim, "Isolation and identification of dermatophytes from infected camels," Sudan J. Vet Res., vol. 25, pp. 94-53, 2010.

[7] D. C. Blood, O. M. Radostits, J. A. Henderson, J. H. Arundel and C. C. Gay, Veterinary Medicine, $6^{\text {th }}$ ed. London: Bailliere and Tindal, 1983.

[8] W. R. Kelly, Veterinary Clinical Diagnosis, 3rd ed. London: Bailliere and Tindal, 1984.

[9] M. M. Maslen, "Human cases of cattle ringworm due to Trichophyton verrucosum in Victoria, Australia," Australian J. Dermatol., vol. 42, pp. 1-4, 2000.

[10] P. X. Ming, Y. L. Ti, and G. S. Bulmer, "Outbreak of Trichophyton verrucosum in China transmitted from cows to humans," Mycopathologia, vol. 161, pp. 225-228, 2006.

[11] S. M. Alghalibi, "Prevalence of dermatomycosis in Sana'a City, Yemen,”Yem. Sci. J., vol. 4, pp. 1-4, 2003.

[12] N. Baghza, A. Mahmoud, and S. Alghalibi, "Mycological and physiological studies on fungi associated with human skin diseases," Yemeni J. Biol. Sci., vol. 8, pp. 59-67, 2012.

[13] H. A. Golah, A. S. Khalel, J. M. Khaled, and K. Shine, "Antifungal susceptibility of dermatophytes isolated from domestic calves in Thamar, Yemen," J. Anim. Vet. Adv., vol. 11, pp. 4544-4548, 2012.

[14] S. A. Shinkafi, and S. B. Manga, "Isolation of dermatophytes and screening of selected medicinal plants used in the treatment of dermatophytoses," I. J. M. R., vol. 2, pp. 40-48, 2001.

[15] L. L. Hungerford, C. L. Campbell, and A. R. Smith, Veterinary Mycology Laboratory Manual. Ames.: Iowa State University Press, 1998

[16] M. A. Klich, Identification of Common Aspergillus Species. United State Department of Agriculture Research Service, Southern Regional Research Center. New Orleans, Louisiana USA, 2002.
[17] A. Chatterjee, P, Chakraborty, D. Chattopadhyay, and D. W. Sengupta, "Isolation of Trichophyton schoenleinii from a camel,” Ind. J. Anim. Hlth., vol. 17, pp. 79-81, 1978.

[18] R. Cecchi, S Paoli, A. Giomi, R. Rossetti, "Favus due to Trichophyton schoenleinii in a patient with metastatic bronchial carcinoma," Br. J. Dermatol., vol. 148, pp. 1057, 2003.

[19] A. Khaled, L B. Mbarek, M, Kharfi, et al., "Tinea capitis favosa due to Trichophyton schoenleinii," Acta Dermatovenerol. Alp. Panonica Adriat, vol. 16, pp. 34-46. 2007

[20] B. Raszeja-Kotelba, Z. Adamski, A. Pawlowicz, "A case of tinea favosa capitis caused by Trichophyton schoenleinii," Przegl Dermatol., vol. 80, pp. 518-524, 1993.

[21] E. I. Nweze, "Etiology of dermatophytes among the children in Eastern Nigeria," J. Med. Myco., vol. 39, pp. 180-184, 2001.

[22] H. H. Arslan, G. F. Yarim, O. Yavuz, and B. Bas, "Positive effects of attenuated Trichophyton verrucosum strain administration in treatment of the bovine trichophytosis," Revue Méd. Vét., vol. 158, pp. 509-513, 2007.

[23] A. M. Abdulaziz, S. A. Osman, and E. M. A. Saeed, "An outbreak of dermatophytosis in camels (Camelus dromedaríus) at Qassim Region, Central of Saudi Arabia,” J. Appl. Anim. Res., vol. 44, pp. 126-129, 2016.

[24] M. A. Mofaddal, Dominant Species of Dermatophytes in Yemeni Patients in Sana'a Governorate, MSc thesis. Yemen: Sana'a University, 2004.

[25] G. A. Vena, p. Chieco, F. Posa, A. Garofalo, A. Bosco, N. Cassano, "Epidemiology of derrmatophytoses: retrospective analysis from 2005 to 2010 and comparison with previous data from 1975," New Microbiol., vol. 35, pp. 207-213, 2012.

[26] N. Mederle, G. Dărăbus, S. Morariu, I. Oprescu, D. Indre, and A. Balint, "Epidemiological aspects in human and cat microsporia," Lucrări Stiintifice Medicină Veterinară, vol. 43, pp. 61-63, 2010.

[27] Y. Sakuragi, Y. Sawada,, Y. Hara, et al., "Increased circulating Th17 cell in a patient with tinea capitis caused by Microsporum canis," Allergol Int., vol. 65, pp. 215-216, 2016.

[28] F. C. Tuteja, N. V. Patil, S. D. Narnaware, G. Nagarajan, and S. S. Dahiya, "Camel dermal mycoses caused by dermatophytes," J. Camel Pract. Res., vol. 20, pp. 157-165, 2013.

[29] C. Rene, L. Ferreiro, L. and G. Jacques, "Dermatophytoses in Animals," Mycopathologia, vol. 166, pp. 385-405, 2008. 